\title{
Critical slowing down and the gradient flow coupling in the Schrödinger functional
}

DESY 13-191

$H U-E P-13 / 54$

SFB/CPP-13-77

\section{Patrick Fritzsch}

Humboldt-Universität, Institut für Physik, Newtonstr. 15, 12489 Berlin, Germany

E-mail: fritzsch@physik.hu-berlin.de

\section{Alberto Ramos \\ NIC, DESY, Platanenallee 6, 15738 Zeuthen, Germany \\ E-mail: alberto.ramos@desy.de}

\section{Felix Stollenwerk*}

Humboldt-Universität, Institut für Physik, Newtonstr. 15, 12489 Berlin, Germany

E-mail: felix.stollenwerk@physik.hu-berlin.de

\begin{abstract}
We study the sensitivity of the gradient flow coupling to sectors of different topological charge and its implications in practical situations. Furthermore, we investigate an alternative definition of the running coupling that is expected to be less sensitive to the problems of the HMC algorithm to efficiently sample all topological sectors.
\end{abstract}

31st International Symposium on Lattice Field Theory LATTICE 2013

July 29 - August 3, 2013

Mainz, Germany

${ }^{*}$ Speaker. 


\section{Introduction}

The gradient (or Wilson) flow in Yang-Mills theory is defined by the non-linear equation [1]

$$
\frac{d B_{\mu}(x, t)}{d t}=D_{v} G_{v \mu}(x, t), \quad B_{\mu}(x, 0)=A_{\mu}(x),
$$

where $t$ is the flow time and $G_{\mu v}=\partial_{\mu} B_{v}-\partial_{v} B_{\mu}+\left[B_{\mu}, B_{v}\right]$. Due to

$$
D_{v} G_{v \mu} \sim-\frac{\delta S_{\mathrm{YM}}[B]}{\delta B_{\mu}}
$$

the flow effectively constitutes a smoothing of the gauge field over a range $\sqrt{8 t}$ and drives it towards a local minimum of the action. One remarkable feature of this procedure is the fact that the gauge field $B_{\mu}$ at $t>0$ is renormalized, rendering expectation values of local, gauge-invariant quantities like the energy density

$$
\langle E(t)\rangle=\frac{1}{4}\left\langle G_{\mu \nu}^{a}(t) G_{\mu v}^{a}(t)\right\rangle
$$

finite [1]. This can be used to define a non-perturbative gradient flow (GF) coupling in terms of the energy density at positive flow time [1] at a renormalization scale $\mu=1 / \sqrt{8 t}$. In order to study the running coupling in asymptotically free theories such as QCD, finite size scaling is applied, i.e., the renormalization scale runs with the size $L$ of the box,

$$
\mu=\frac{1}{\sqrt{8 t}}=\frac{1}{c L},
$$

where $c$ represents the fraction of the box over which the gauge field is smoothed. This approach was implemented in a periodic box [2] and in the Schrödinger functional (SF) [3], where the GF coupling is defined as

$$
\bar{g}_{\mathrm{GF}}^{2}(L):=\left.\mathscr{N}^{-1} \cdot t^{2}\left\langle E\left(t, x_{0}\right)\right\rangle\right|_{t=c^{2} L^{2} / 8} ^{x_{0}=T / 2} .
$$

The normalization factor $\mathscr{N}^{-1}$ in (1.5) ensures $\bar{g}_{\mathrm{GF}}^{2}=g_{0}^{2}+\mathscr{O}\left(g_{0}^{4}\right)$. Note that as the SF breaks translational invariance in time direction, the energy density becomes explicitly dependent ${ }^{1}$ on $x_{0}$.

In [3], the GF coupling has been investigated on the lattice in view of numerical costs and cutoff effects for ensembles of $N_{f}=2$ simulations at a line of constant physics, defined by a constant value of the SF coupling, which corresponds to $L \sim 0.4 \mathrm{fm}$. Smoothing fractions in the range $c \in[0.3,0.5]$ turn out to be convenient, as their use leads to high statistical precision at affordable cost and modest cutoff effects. However, at the considered physical volume, the path integral is largely dominated by the trivial topological sector $(Q=0)$ and contributions from other sectors can be considered negligible.

In contrast, at larger volumes like $L \sim 0.8 \mathrm{fm}$, sectors of non-vanishing topological charge are expected to contribute, which brings up the question about the well-known problems of topology freezing and critical slowing down [4]. The aim of the present work is to investigate whether and how the determination of the gradient flow coupling is affected by these phenomena.

\footnotetext{
${ }^{1}$ Boundary fields and fermionic phase angle of the SF need to be specified as well.
} 


\begin{tabular}{c|c|c|c}
$L / a$ & $\beta$ & $N_{\mathrm{ms}}$ & MDUs \\
\hline 8 & 5.9032 & 80000 & 6 \\
12 & 6.1410 & 80000 & 6 \\
16 & 6.3413 & 40000 & 6 \\
20 & 6.5119 & 15000 & 12 \\
24 & 6.6552 & 7000 & 12
\end{tabular}

Table 1: Parameters of the numerical simulations. $N_{\mathrm{ms}}$ is the amount of measurements and the last column shows the number of molecular dynamic units between two consecutive measurements.

\begin{tabular}{c|c|c}
$L / a$ & $c=0.3$ & $c=0.5$ \\
\hline 8 & $98.66(8)$ & $98.94(8)$ \\
12 & $98.19(20)$ & $98.45(19)$ \\
16 & $98.46(62)$ & $98.56(61)$ \\
20 & $* 99.91(3)$ & $* 99.96(2)$ \\
24 & $* 99.52(36)$ & $* 99.54(36)$
\end{tabular}

Table 2: Percent of configurations with topological charge $Q \leq 0.5$. The values denoted with an asterisk are biased.

\section{Numerical simulations}

In order to be able to produce large statistics, we perform simulations in pure SU(3) YangMills theory with the Wilson gauge action. We choose SF boundary conditions with zero boundary gauge fields, and fix the physical volume in terms of the Sommer scale:

$$
L=r_{0} / 0.563 \sim 0.8 \mathrm{fm} .
$$

For lattice sizes of $L / a=8,12,16,20,24$, we simulate along the line of constant physics defined by (2.1). The corresponding bare couplings $\left(\beta=6 / g_{0}^{2}\right)$ are determined using [5]

$$
\log \left(a / r_{0}\right)=-1.6804-1.7331(\beta-6)+0.7849(\beta-6)^{2}-0.4428(\beta-6)^{3}
$$

and can be found along with other parameters of the simulation in Tab. 1. We use the HMC of the openQCD package [6] and the 2-loop value of $c_{t}$ for $\mathscr{O}(a)$ improvement [7]. Each produced configuration is evolved by integration of the flow equation (1.1), including flow times $t$ corresponding to $c=0.3,0.5$. Afterwards, on the smoothed configurations the topological charge,

$$
Q(t)=\frac{1}{16 \pi^{2}} \sum_{x} G_{\mu v}(x, t) \tilde{G}_{\mu v}(x, t),
$$

and the GF coupling are measured using the clover discretisation for the field strength.

\section{Results}

\subsection{Distribution of the topological charge}

Histories of the topological charge are shown in Fig. 1 for $c=0.3$. For lattices up to $L / a=16$, one observes that non-trivial configurations appear to cluster more and more as the lattice gets finer. This goes together with the increasing integrated autocorrelation time displayed in Fig. 2. For the largest lattices $L / a=20,24$, configurations from non-trivial sectors appear less often, see Tab. 2 . However, the autocorrelations of $Q$ are obviously largely underestimated (cf. Fig. 2), from which we infer that the current statistics is not sufficiently large to sample the topological sectors correctly. Note that throughout this work, statistical errors were computed using the $\Gamma$-method $[8,9]$. 

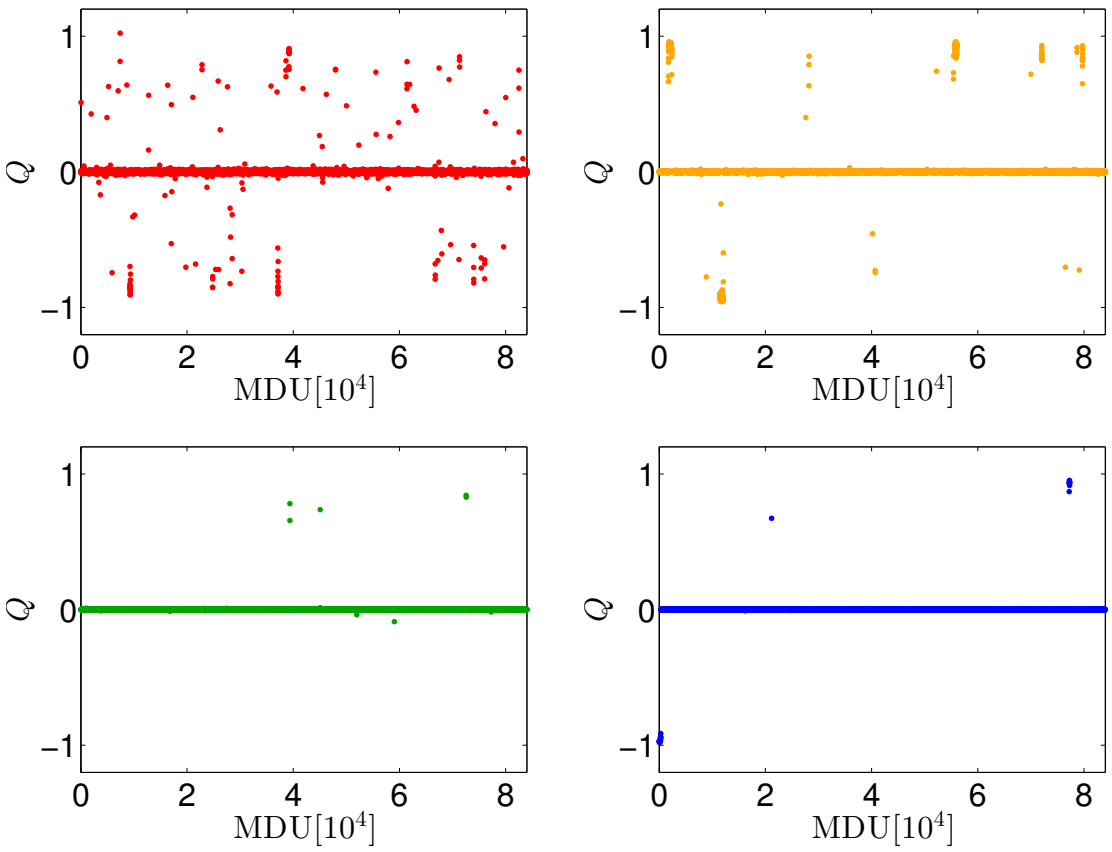

Figure 1: Histories (in excerpts) of $Q$, for $c=0.3$. The plots in red, orange, green and blue correspond to $L / a=12,16,20,24$, respectively.
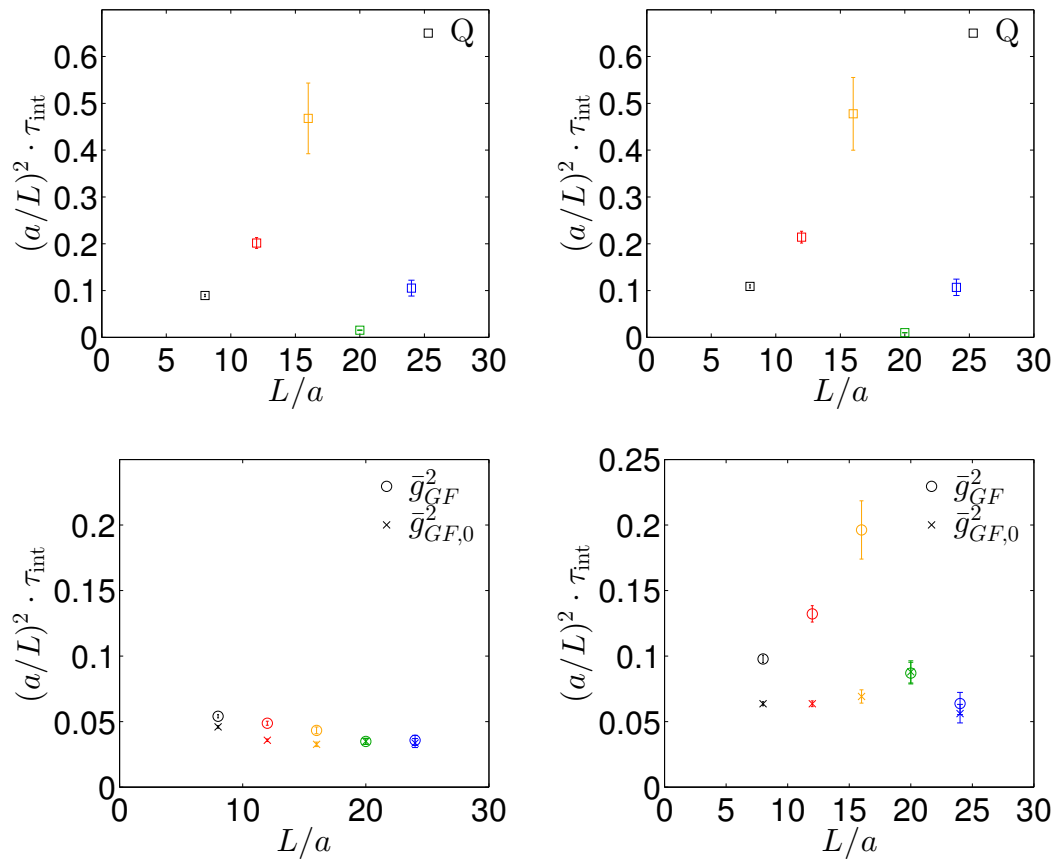

Figure 2: Integrated autocorrelation time of the topological charge $Q$ (top) and the (modified) gradient flow coupling (bottom), multiplied by $(a / L)^{2}$ and in units of 2 MDU. The left panel corresponds to $c=0.3$, the right one to $c=0.5$. 

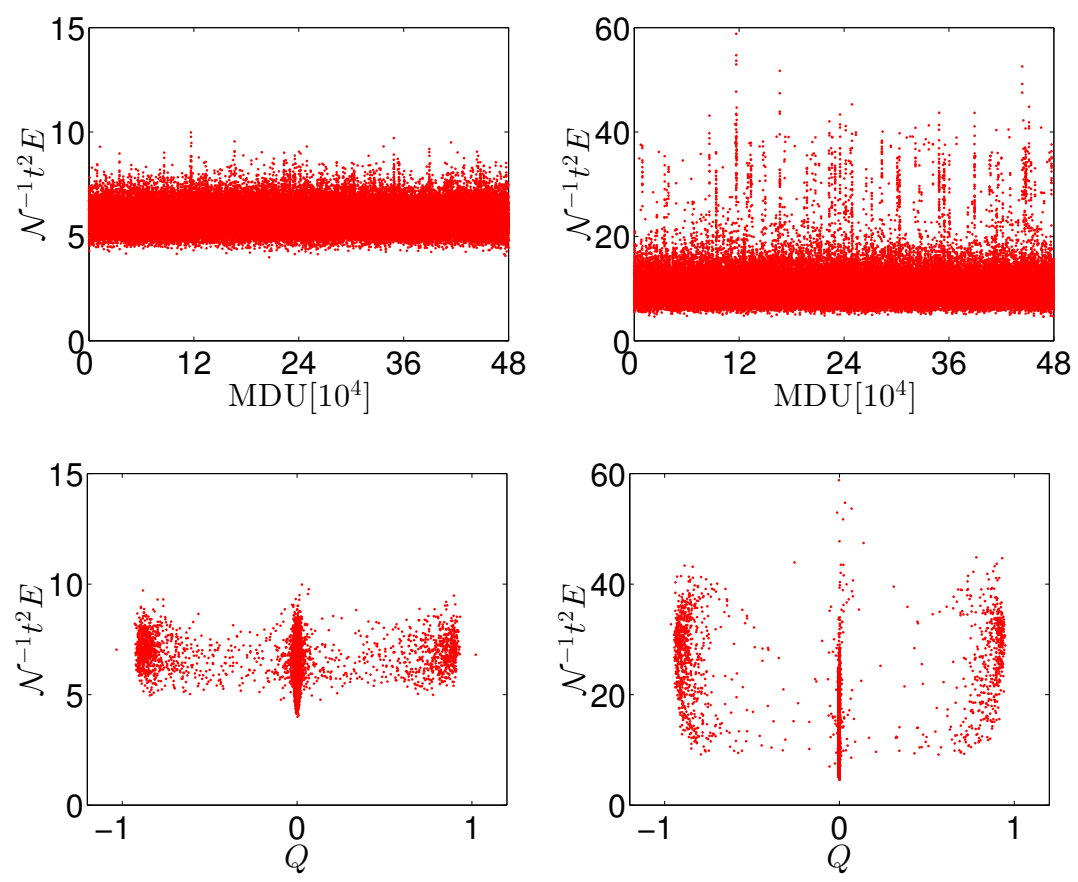

Figure 3: Top: Histories of $\bar{g}_{\mathrm{GF}}^{2}$. Bottom: Correlation of $\bar{g}_{\mathrm{GF}}^{2}$ and $Q$. All plots are shown exemplarily for $L / a=12$. The left panel corresponds to $c=0.3$, the right one to $c=0.5$.

\subsection{Correlation of gradient flow coupling and topological charge}

The histories of the quantity $\mathscr{N}^{-1} t^{2} E$, whose expectation value is the gradient flow coupling $\bar{g}_{\mathrm{GF}}^{2}$, exhibit a certain amount of large values, see Fig. 3. This phenomenon is more pronounced the larger $c$ is chosen, and goes along with an increasing correlation between the gradient flow coupling and the topological charge. In particular, the large values of $\mathscr{N}^{-1} t^{2} E$ stem to a high amount from configurations of non-vanishing topological charge. In turn, the correct sampling of the topological sectors becomes a necessity in order to obtain correct results. However, as we have seen in sec. 3.1, this requirement is not fulfilled for the two largest lattices, which means that the results for $\bar{g}_{\mathrm{GF}}^{2}$ on these lattices are biased.

\subsection{The modified GF coupling}

In order to assess the impact of the non-trivial topological sectors and their insufficient sampling on the determination of $\bar{g}_{\mathrm{GF}}^{2}$, we consider a modified GF coupling, which has the same perturbative expansion but takes into account only gauge configurations from the trivial sector:

$$
\bar{g}_{\mathrm{GF}, 0}^{2}=\left.\mathscr{N}^{-1} t^{2} \frac{\left\langle E(t) \delta_{Q, 0}\right\rangle}{\left\langle\delta_{Q, 0}\right\rangle}\right|_{t=c^{2} L^{2} / 8} .
$$

On the lattice, where we have non-integer values of $Q$, all configurations with $|Q| \leq \varepsilon(\varepsilon=0.1,0.2$, $\ldots, 0.5)$ are considered to belong to the trivial sector, i.e., we replace $\delta_{Q, 0} \rightarrow \Theta(Q+\varepsilon) \Theta(\varepsilon-Q)$ in (3.1). The results for $L / a=12$ can be seen in Fig. 4. The contributions from non-trivial sectors do make a difference, and the effect is stronger for large $c$ due to the larger correlation discussed in sec. 3.2. Moreover, we see that the particular choice of $\varepsilon$ has no big influence on the modified 

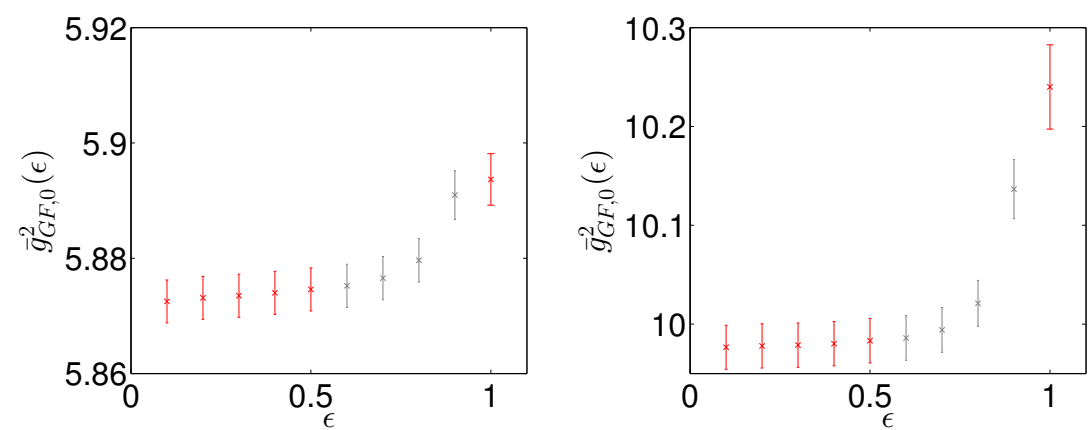

Figure 4: Modified gradient flow coupling $\bar{g}_{\mathrm{GF}, 0}^{2}$ for $L / a=12$ against the range $\varepsilon$, where $\varepsilon \leq 0.5$ can serve as definition of the trivial sector on the lattice. Results for larger $\varepsilon$ take into account configurations which are considered non-trivial, but are nevertheless shown in gray for completeness. The point at $\varepsilon=1$ corresponds to the original definition $\bar{g}_{\mathrm{GF}}^{2}$ of the gradient flow coupling. Left: $c=0.3$. Right: $c=0.5$.

gradient flow coupling. We use $\varepsilon=0.5$ in the following. In Fig. 2, we compare the integrated autocorrelation time for the gradient flow coupling in its original and modified form. We find that the original coupling is affected by the bad sampling towards the continuum, whereas the modified coupling does suffer less severely from critical slowing down and shows the expected $\sim 1 / a^{2}$ behavior. In that sense, the modified gradient flow coupling can be considered to be safer.

\subsection{Results and continuum limit}

The full set of results for the two couplings $\bar{g}_{\mathrm{GF}}^{2}$ and $\bar{g}_{\mathrm{GF}, 0}^{2}$ is listed in Tab. 3. On the coarser lattices $(L / a=8,12,16)$, the simulations show a clear difference between the two definitions. This suggests that in the studied volume $(L \sim 0.8 \mathrm{fm})$, topologically non-trivial configurations play a role in accurately determining the value of $\bar{g}_{\mathrm{GF}}^{2}$. On the two finer lattices $(L / a=20,24)$, we do not observe a difference due to the critical slowing down that affects the determination of the original coupling.

Since the results for $\bar{g}_{\mathrm{GF}}^{2}$ on the finer lattices $(L / a=20,24)$ are biased, we conduct the continuum extrapolation only for $\bar{g}_{\mathrm{GF}, 0}^{2}$. To compare data of different lattice spacings we have to take into account an additional error being introduced by the way the physical volume was fixed, Eq. $(2.2)^{2}$. This uncertainty turns out to be larger than the statistical errors (see Tab. 3). We find that the data with $L / a>8$ is well described by a fit linear in $(a / L)^{2}$, see Fig. 5 .

\section{Summary \& Conclusions}

We investigated the gradient flow coupling in pure SU(3) Yang-Mills theory in a volume of $L \sim 0.8 \mathrm{fm}$. We find a significant correlation between the coupling and the topological charge, which increases in strength with the smoothing fraction $c$. Simulations suffering from a bad sampling of topological sectors and critical slowing down would lead to a biased determination of $\bar{g}_{\mathrm{GF}}^{2}$ in the continuum. We propose an alternative definition for the coupling $\left(\bar{g}_{\mathrm{GF}, 0}^{2}\right)$, which takes into account only the trivial topological sector, and show that its determination is not affected by the

\footnotetext{
${ }^{2}$ The error on $a / r_{0}(\beta)$ depends on $\beta$, but for simplicity we propagate its maximum value of $1 \%$ globally [5].
} 

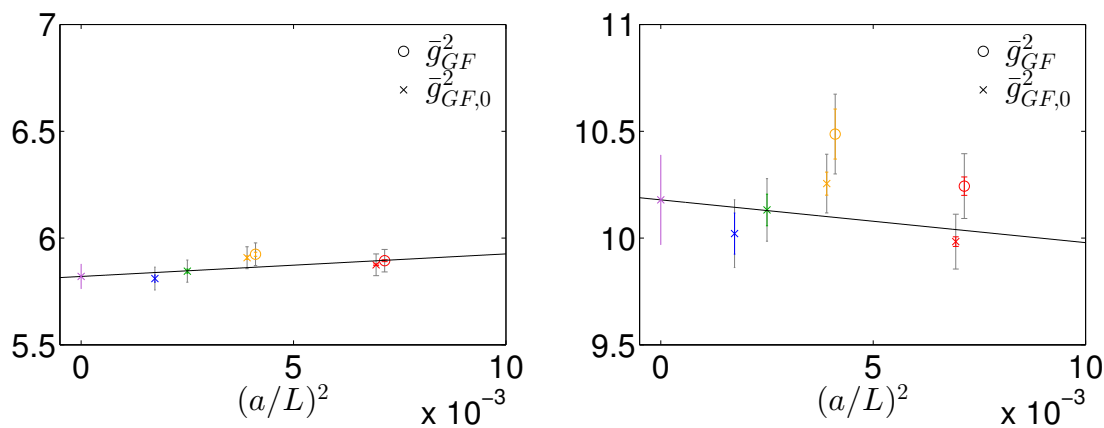

Figure 5: Continuum extrapolation of the modified gradient flow coupling (crosses). The unbiased data for the gradient flow coupling is also shown (open circles), the points being slightly shifted to the right for convenience. Statistical errors are displayed in color, errors from the line of constant physics in gray. Left: $c=0.3$. Right: $c=0.5$.

\begin{tabular}{c|c|c|c|c}
\multirow{2}{*}{$L / a$} & \multicolumn{2}{|c|}{$c=0.3$} & \multicolumn{2}{c}{$c=0.5$} \\
\cline { 2 - 5 } & $\bar{g}_{\mathrm{GF}}^{2}$ & $\bar{g}_{\mathrm{GF}, 0}^{2}$ & $\bar{g}_{\mathrm{GF}}^{2}$ & $\bar{g}_{\mathrm{GF}, 0}^{2}$ \\
\hline 8 & $5.647(3)(52)$ & $5.631(3)(51)$ & $9.313(21)(146)$ & $9.140(14)(126)$ \\
12 & $5.894(5)(52)$ & $5.875(4)(51)$ & $10.243(43)(146)$ & $9.983(23)(126)$ \\
16 & $5.924(10)(52)$ & $5.908(8)(51)$ & $10.487(117)(146)$ & $10.255(54)(126)$ \\
20 & $* 5.845(10)(52)$ & $5.845(10)(51)$ & $* 10.135(74)(146)$ & $10.132(74)(126)$ \\
24 & $* 5.818(28)(52)$ & $5.810(17)(51)$ & $* 10.128(123)(146)$ & $10.021(96)(126)$ \\
\hline$\infty$ & $* 5.818(62)$ & $5.820(58)$ & $* 10.156(258)$ & $10.179(210)$
\end{tabular}

Table 3: Results for the (modified) gradient flow coupling and its continuum extrapolation. The first error is statistical, the second one stems from fixing the physical volume. Biased values are denoted with an asterisk.

bad topology sampling in the studied volume. We think that the alternative definition might be advantageous for the determination of the running coupling in intermediate volumes.

\section{References}

[1] M. Lüscher. JHEP 1008 (2010) 071, [arXiv:1006.4518];

M. Lüscher and P. Weisz. JHEP 1102 (2011) 051, [arXiv: 1101 . 0963 ]

[2] Z. Fodor et al. JHEP 1211 (2012) 007, [arXiv:1208.1051]

[3] P. Fritzsch, A. Ramos. JHEP 1310 (2013) 008, [arXiv: 1301 . 4388 ]

[4] S. Schaefer et al. Nucl. Phys. B 845, 93 (2011) [arXiv: hep-lat/1009.5228]

[5] S. Necco, R. Sommer. Nucl. Phys. B622 (2002) 328-346, [arXiv: hep-lat/0108008 ]

[6] M. Lüscher, S. Schaefer. JHEP 1107 (2011) 036, [arXiv:1105.4749]

[7] A. Bode, P. Weisz, U. Wolff. Nucl. Phys. B576 (2000) 517-539, [arXiv: hep-lat/9911018 ]

[8] N. Madras, A. D. Sokal. J.Statist.Phys. 50 (1988) 109-186

[9] U. Wolff. Comput.Phys.Commun. 156 (2004) 143-153, [arXiv: hep-lat/0306017] 